\title{
Desenvolvimento vegetativo e produção de óleo essencial de patchouli (Pogostemon cablin (Blanco) Benth.) após a aplicação de ácido giberélico e extrato de alga marinha
}

STORCK, R.C. ${ }^{1 *}$; DESCHAMPS, C. ${ }^{1}$; MÓGOR, A.F.'; COCCÔ, L.C..; SCHEER, A.P. ${ }^{2}$; YAMAMOTO, C.I. ${ }^{2}$ ${ }^{1}$ Universidade Federal do Paraná, Rua dos Funcionários $n^{\circ}$ 1540, CEP 80035-050 Curitiba, Paraná - Brasil *rafaellencstorck@yahoo.com.br, ${ }^{2}$ Universidade Federal do Paraná, Centro Politécnico, CEP: 81530-990 Curitiba, Paraná - Brasil

RESUMO: Pogostemon cablin (Blanco) Benth., comumente conhecido como patchouli, é uma espécie aromática utilizada pela indústria de perfumes devido a fragrância amadeirada e propriedade fixadora do óleo essencial. Fatores que afetam o teor dos constituintes do óleo essencial devem ser avaliados visando obter matéria prima de melhor qualidade. O experimento foi conduzido em casa de vegetação com delineamento inteiramente casualizado em esquema fatorial $2 \times 3$ e 4 repetições, sendo dois níveis de giberelina $\left(0\right.$ e $\left.200 \mathrm{mg} \mathrm{L}^{-1}\right)$ e três níveis de extrato de alga marinha $\left(0,15\right.$ e $\left.30 \mathrm{mg} \mathrm{L}^{-1}\right)$. A aplicação dos reguladores vegetais foi realizada 30 dias após o plantio das mudas, e a avaliação do experimento deu-se 45 dias após a aplicação dos tratamentos. Não houve diferença significativa para a altura das plantas, a concentração de extrato de alga marinha de $15 \mathrm{mg} \mathrm{L}^{-1}$ promoveu aumento no número de folhas em relação à testemunha e a concentração de $30 \mathrm{mg} \mathrm{L}^{-1}$ promoveu decréscimo. Em relação à área foliar e ao número de folhas houve relação inversa para os níveis de extrato de alga marinha, onde o tratamento com maior concentração do regulador resultou em aumento da área foliar e redução do número de folhas. O teor de óleo essencial foi superior após a aplicação de $15 \mathrm{mg} \mathrm{L}^{-1} \mathrm{de}$ extrato alga marinha isoladamente ou combinado com $\mathrm{GA}_{3}$. A produtividade do óleo essencial também aumentou com a aplicação $15 \mathrm{mg} \mathrm{L}^{-1}$ de extrato de alga marinha e quando utilizado somente $\mathrm{GA}_{3}$. A utilização de extrato de alga marinha aumentou a concentração de patchoulol no óleo essencial.

Palavras-chave: metabolismo secundário, reguladores vegetais, espécie aromática

ABSTRACT: Development and essential oil production of Pogostemon cablin (Blanco) Benth., patchouli after applying gibberellic acid and seaweed extract. Pogostemon cablin (Blanco) Benth., commonly known as patchouli, is an aromatic species used by the perfume industry due to its woody fragrance and the fixative characteristic of its essential oil. Factors affecting the content of the constituents of essential oils should be evaluated in order to obtain raw materials with better quality. The experiment was carried out in a greenhouse in a completely randomized $2 \times 3$ factorial arrangement and four replications, with two levels of gibberellin ( 0 and $\left.200 \mathrm{mg} \mathrm{L}^{-1}\right)$ and three levels of seaweed extract $\left(0,15\right.$ and $\left.30 \mathrm{mg} \mathrm{L}^{-1}\right)$. Plant regulators were applied thirty days after planting the seedlings; the experiment was evaluated forty-five days after the treatments. There was no significant difference in plant height; the $15 \mathrm{mg} \mathrm{L}^{-1}$ concentration of seaweed extract promoted an increase in the number of leaves in relation to the control; and the $30 \mathrm{mg} \mathrm{L}^{-1}$ concentration promoted a decrease. In relation to leaf area and leaf number, there was an inverse relationship for the seaweed extract levels, in which the treatment with higher a concentration of the regulator resulted in an increased leaf area, reducing the number of leaves. The essential oil content was higher after applying $15 \mathrm{mg} \mathrm{L}^{-1}$ of seaweed extract alone or combined with $\mathrm{GA}_{3}$. The yield of essential oil also increased by applying $15 \mathrm{mg} \mathrm{L}^{-1}$ of seaweed extract and when only $\mathrm{GA}_{3}$ was used. The use of seaweed extract increased the patchoulol concentration in the essential oil.

Keywords: secondary metabolism, plant regulators, aromatic species

Recebido para publicação em 23/09/2010

Aceito para publicação em 05/12/2012

Rev. Bras. PI. Med., Campinas, v.15, n.3, p.391-396, 2013. 


\section{INTRODUÇÃO}

Pogostemon cablin (Blanco) Benth., popularmente conhecido como patchouli, é uma erva de hábito perene com altura variável entre 0,6 a $1,0 \mathrm{~m}$ e filotaxia oposta com folhas ovaladas, adaptando-se em ambientes semi-sombreados (Epagri, 2004).

O óleo essencial produzido por esta espécie possui atividades farmacológicas, sendo as propriedades terapêuticas diretamente relacionadas à qualidade e a quantidade deste. Devido à fragrância amadeirada e capacidade de fixação à pele é também amplamente utilizado por indústrias de cosméticos e de perfumarias (Hu et al., 2005; Sugimura et al., 2005; Zhao et al., 2005).

Crouch \& Van Staden (1993) relatam a utilização de algas marinhas e formulações a base destas, durante muitos anos em práticas agrícolas. Sabe-se que algas marinhas possuem alguns hormônios vegetais em sua composição. Ergün et al. (2002) demonstraram a presença de ácido indol-3-acético (IAA), ácido giberélico (GA3), ácido abscísico (ABA) e citocinina (zeatina) em algumas espécies de liquens. Zhang \& Ervin (2004), por meio de testes de ELISA (enzyme-linked immunosorbent assay), descreveram a presença de zeatina ribosideo (66 $\mu \mathrm{g} g-1)$ e de isopentinil adenina (iPA) (4 $\mu \mathrm{g} \mathrm{g-1)}$ no extrato da alga parda Ascophyllum nodosum (L.) Le Jolis.

Citocininas e giberelinas são capazes de promover diversos efeitos fisiológicos nas plantas. Entretanto, o uso de reguladores vegetais pode alterar o comportamento de algumas plantas por meio da produtividade bem como do metabolismo secundário (Shukla \& Farooqi, 1990).

Quando aplicadas exogenamente, as citocininas podem promover o aumento da produção de óleos essenciais bem como alterações na composição (Stoeva \& llieve, 1997). Storck (2008) demonstrou em plantas de Pogostemon cablin Benth., que a aplicação de giberelina em plantas submetidas à um nível de $54 \%$ de sombreamento resulta em maior teor e produtividade do óleo essencial, bem como promove alterações na composição.

Shukla \& Farooqi (1990) sugerem que a aplicação de giberilinas e citocininas podem otimizar o teor de óleos essenciais. Conforme observado em plantas de Artemisia annua L., a aplicação de GA3 resultou em aumento da produção de artemisina e a aplicação de cinetina aumentou o rendimento do óleo essencial (Farooqi et al., 1996).

Este trabalho teve como objetivo avaliar o desenvolvimento vegetativo, o teor, a produtividade e a composição do óleo essencial de Pogostemon cablin (Blanco) Benth. após aplicações de extrato de alga marinha Ascophyllum nodosum e de GA3, submetido a $54 \%$ de sombreamento.

\section{MATERIAL E MÉTODO}

O presente trabalho foi conduzido em condições de casa-de-vegetação, no Departamento de Fitotecnia e Fitossanitarismo da Universidade Federal do Paraná (Curitiba - PR), durante os meses de agosto a novembro de 2007. O material vegetal utilizado constitui-se de plantas de Pogostemon cablin (Blanco) Benth., cedidas pela empresa Hérbia Óleos essenciais Ltda. situada no município de Joinville - SC. Deste material vegetal foram obtidas mudas por meio de estaquia $(6 \mathrm{~cm}$ de comprimento com um par de folhas reduzidas à metade), as quais foram utilizadas na realização deste experimento.

O delineamento experimental utilizado foi inteiramente casualizado em esquema fatorial $2 \times 3$ com 4 repetições, sendo dois níveis de giberelina $(0$ e $\left.200 \mathrm{mg} \mathrm{L}^{-1}\right)$ e três níveis de extrato de alga marinha $\left(0,15\right.$ e $\left.30 \mathrm{mg} \mathrm{L}^{-1}\right)$, cada qual com 4 vasos $(17 \mathrm{~cm}$ de altura e $23 \mathrm{~cm}$ de diâmetro) e capacidade para aproximadamente $6 \mathrm{Kg}$ de substrato terra.

$\mathrm{O}$ substrato apresentou as seguintes características: 4,30 $\mathrm{CaCl}_{2} ; 5,20 \mathrm{SMP} ; 3,60 \mathrm{Al}^{+3}$ $\left(\mathrm{cmol}_{\mathrm{c}} \mathrm{dm}^{-3}\right) ; 9,00 \mathrm{H}+\mathrm{Al}^{+3}\left(\mathrm{cmol}_{\mathrm{c}} \mathrm{dm}^{3}\right) ; 1,60 \mathrm{Ca}^{+2}$ $\left(\mathrm{cmol}_{\mathrm{c}} \mathrm{dm}^{3}\right) ; 0,80 \mathrm{Mg}^{+2}\left(\mathrm{cmol}_{\mathrm{c}} \mathrm{dm}^{3}\right) ; 0,07 \mathrm{~K}^{+}\left(\mathrm{cmol}_{\mathrm{C}}\right.$ $\left.\mathrm{dm}^{3}\right) ; 2,47 \mathrm{SB}\left(\mathrm{cmol}_{\mathrm{c}} \mathrm{dm}^{3}\right) ; 11,47 \mathrm{~T}\left(\mathrm{cmol}_{\mathrm{c}} \mathrm{dm}^{3}\right) ; 5,70$ $\mathrm{P}\left(\mathrm{mg} \mathrm{dm}^{3}\right) ; 41,4 \mathrm{C}\left(\mathrm{g} \mathrm{dm}^{3}\right) ; 22 \mathrm{~V}(\%) ; 59 \mathrm{~m}(\%) ; 2,0$ $\mathrm{Ca} / \mathrm{Mg}$ e 400,0 argila (g Kg).

Para a correção do $\mathrm{pH}$ do substrato, foi incorporado 8,3 toneladas ha-1 de calcário (Raij et al., 1996). Ainda conforme recomendação destes autores, foi incorporado $30 \mathrm{Kg}$ ha ${ }^{-1}$ de $\mathrm{N}, 130 \mathrm{Kg}$ ha ${ }^{-1}$ de $\mathrm{P}_{2} \mathrm{O}_{5}$ e $110 \mathrm{Kg}$ ha ${ }^{-1}$ de $\mathrm{K}_{2} \mathrm{O}$.

O material vegetal foi então submetido ao sombreamento de $54 \%$ utilizando-se Sombrite ${ }^{\circledR}$, e a aplicação de Pro Gibb ${ }^{\circledR}$ 10\% GA3 $(200$ mg $\left.\mathrm{L}^{-1}\right)$ (Storck, 2008) e extrato da alga marinha Ascophyllum nodosum (L.) Le Jolis como fonte de citocinia $0,001 \%$ (15 e $30 \mathrm{mg} \mathrm{L}^{-1}$ ). A aplicação dos reguladores foi realizada com equipamento de pulverização pressurizado por $\mathrm{CO} 2 \mathrm{com}$ vazão e pressão constante (50 Psi), 30 dias após a implantação do experimento. Para determinar com maior precisão o nível de sombreamento, foram feitas medições utilizando-se o aparelho porômetro, modelo LICOR 1600, em dias ensolarados e nublados.

O desenvolvimento vegetativo do material foi avaliado aos 75 dias após a implantação do experimento (45 dias após aplicação dos reguladores) a partir da determinação da área foliar, altura $(\mathrm{cm})$, número de folhas e número de ramos.

Para a extração do óleo essencial utilizouse $30 \mathrm{~g}$ tecido foliar com aproximadamente $20 \%$ de umidade para hidrodestilação durante 5 horas em aparelho graduado Clevenger e balão volumétrico de $2 \mathrm{~L}$, conforme testes preliminares realizados em

Rev. Bras. PI. Med., Campinas, v.15, n.3, p.391-396, 2013. 
laboratório com a mesma espécie.

Para calcular o teor do óleo essencial o volume retirado do Clevenger foi quantificado com micropipetas de precisão e corrigido com base de massa seca foliar de cada tratamento. Para quantificar a produtividade do óleo essencial estimou-se a biomassa de folhas por hectare com base nos valores obtidos no experimento, sabendose que a cultura do patchouli comporta 10000 plantas por hectare. Com base nesses valores obteve-se o volume de óleo essencial em microlitros para cada grama de massa seca produzida por hectare. A partir destes valores o volume de óleo essencial por grama de massa seca foi convertido em litros e multiplicado pela biomassa seca de folhas por hectare, gerando desta forma os valores de produtividade do óleo essencial em litros por hectare $\left(\mathrm{L} \mathrm{ha}^{-1}\right)$.

As amostras do óleo essencial foram analisadas por meio de cromatografia a gás acoplada a espectrometria de massa (CG/EM), utilizando-se cromatógrafo gasoso Varian, modelo CP 3800, com detector FID (CG_FID), coluna capilar Chrompack de sílica fundida CP-SIL 8 CB, 0,25 mm de diâmetro interno, $30 \mathrm{~m}$ de comprimento e 0,25 $\mu \mathrm{m}$ de filme líquido, com temperatura do injetor: $250^{\circ} \mathrm{C}$, split 1:300, volume de amostra injetada: 1,0 $\mu \mathrm{L}$, gás de arraste: hélio $1 \mathrm{~mL}$ min constante, gás de make up: ar sintético, nitrogênio e hidrogênio.

A temperatura do detector FID foi de $300^{\circ} \mathrm{C}$, programação de temperatura do forno: temperatura inicial de $50^{\circ} \mathrm{C}$, elevação de temperatura a $180^{\circ} \mathrm{C}$ na razão de $10^{\circ} \mathrm{C}$ permanecendo por 20 minutos; elevação de temperatura a $200^{\circ} \mathrm{C}$ na razão de $20^{\circ} \mathrm{C}$ permanecendo por 1 minuto. O tempo total da corrida foi de 35 minutos.

As análises de variâncias foram realizadas utilizando-se o programa estatístico ASSISTAT versão 7.4 beta (Silva \& Azevedo, 2006). As variâncias dos tratamentos foram testadas quanto à homogeneidade pelo teste de Bartlett e as médias dos tratamentos comparadas pelo teste de Tukey a $5 \%$ de probabilidade.

\section{RESULTADOS E DISCUSSÃO}

Houve aumento significativo da altura de plantas de Pogostemon cablin após a aplicação de $\mathrm{GA}_{3}$, porém sem efeito do extrato de alga marinha nas concentrações testadas. Farooqi et al. (1993) obtiveram resultados semelhantes com plantas de Artemisia pallens Wall, testando as concentrações de 100, 150 e $200 \mathrm{mg} \mathrm{L}^{-1}$ de $\mathrm{GA}_{3}$, observando aumento da altura das plantas em todas as concentrações. Entretanto, neste trabalho não houve interação significativa entre os fatores extrato de alga marinha e giberelina para as plantas de Pogostemon cablin aos 75 dias de cultivo. Por outro lado, Chaudry \& Khan (2000) verificaram em Cicer arietinum L. que a aplicação de $20 \mathrm{mg} \mathrm{L}^{-1}$ de cinetina e cinetina combinada a $\mathrm{GA}_{3}$ resultam em aumento significativo na altura das plantas a partir dos 45 de cultivo.

A aplicação isolada de $15 \mathrm{mg} \mathrm{L}^{-1}$ extrato de alga marinha promoveu aumento no número de folhas em relação à testemunha e a concentração de $30 \mathrm{mg} \mathrm{L}^{-1}$ promoveu decréscimo. Em relação à área foliar e ao número de folhas, houve relação inversa para os níveis extrato de alga marinha, onde o tratamento que recebeu a maior concentração deste extrato apresentou maior área foliar. Por outro lado, a aplicação de menor concentração de extrato de alga marinha resultou em redução na área foliar.

Após a aplicação da giberelina foi possível observar que quanto maior a concentração de extrato de alga aplicado menor é o número de folhas, e a maior área foliar foi encontrada quando o $\mathrm{GA}_{3}$ foi combinado a aplicação de $15 \mathrm{mg} \mathrm{L}^{-1}$ de extrato de alga marinha.

Em relação ao número de ramos, a aplicação de $200 \mathrm{mg} \mathrm{L}^{-1}$ de $\mathrm{GA}_{3}$ combinado a $30 \mathrm{mg}$ $\mathrm{L}^{-1}$ de extrato de alga marinha e $15 \mathrm{mg} \mathrm{L}^{-1}$ de extrato de alga marinha, foram os que promoveram maiores números de ramos respectivamente.

Para os teores de massa seca de folhas, foi possível observar que a aplicação de extrato de alga marinha em até $15 \mathrm{mg} \mathrm{L}^{-1}$ foi superior em relação ao tratamento com $30 \mathrm{mg} \mathrm{L}^{-1}$. Por outro lado, após a aplicação de $\mathrm{GA}_{3}$ o tratamento com $15 \mathrm{mg}$ $\mathrm{L}^{-1}$ de extrato de alga marinha foi o que promoveu os menores teores de massa seca foliar. Entretanto, a utilização de $15 \mathrm{mg} \mathrm{L}^{-1}$ extrato de alga marinha resultou em aumento nos teores de massa seca de ramos. Após a aplicação de $\mathrm{GA}_{3}$ combinada com extrato de alga marinha, não houve diferença significativa nos teores de massa seca de ramos (Tabela 1).

Houve alteração no teor do óleo essencial em relação aos diferentes reguladores vegetais utilizados (Tabela 2). Observou-se que a aplicação de extrato de alga marinha resultou em aumento do teor até a concentração de $15 \mathrm{mg} \mathrm{L}^{-1}$, sendo que a concentração de $30 \mathrm{mg} \mathrm{L}^{-1}$ resultou em decréscimo. Resultados semelhantes foram obtidos por Scavroni et al. (2006) que observaram redução no teor do óleo essencial de Mentha piperita L. quando tratada com citocinina (BAP) na concentração de $50 \mathrm{mg}$ $\mathrm{L}^{-1}$. O maior teor de óleo essencial nas plantas submetidas a $15 \mathrm{mg} \mathrm{L}^{-1}$ de extrato de alga marinha e a $200 \mathrm{mg} \mathrm{L}^{-1}$ de $\mathrm{GA}_{3}$, pode estar relacionado ao aumento do número de folhas e da massa seca foliar nesses tratamentos, sabendo-se que as estruturas de armazenamento de óleos nessa espécie estão predominantemente presentes nas folhas (Henderson et al., 1970). Da mesma forma,

Rev. Bras. Pl. Med., Campinas, v.15, n.3, p.391-396, 2013. 
TABELA 1. Desenvolvimento vegetativo de Pogostemon cablin aos 75 dias após aplicação de extrato de alga marinha extrato de alga marinha e $200 \mathrm{mg} \mathrm{L}^{-1} \mathrm{GA}_{3}$. Curitiba-PR, 2007.

\begin{tabular}{|c|c|c|c|c|}
\hline \multirow{2}{*}{$\mathrm{GA}_{3}\left(\mathrm{mg} \mathrm{L} \mathrm{L}^{-1}\right)$} & & \multicolumn{3}{|c|}{ Extrato de alga marinha $\left(\mathrm{mg} \mathrm{L}^{-1}\right)$} \\
\hline & & 0 & 15 & 30 \\
\hline \multicolumn{5}{|l|}{ Altura (cm) } \\
\hline 0 & $58,16 \mathrm{~B}$ & $58,50 \mathrm{~ns}$ & $58,00 \mathrm{~ns}$ & $58,00 \mathrm{~ns}$ \\
\hline 200 & $61,25 \mathrm{~A}$ & $63,75 \mathrm{~ns}$ & $62,00 \mathrm{~ns}$ & $58,00 \mathrm{~ns}$ \\
\hline \multicolumn{5}{|c|}{ Número de ramos } \\
\hline 0 & “ & $4,08 \mathrm{Bb}$ & $5,77 \mathrm{Aa}$ & $4,37 \mathrm{Bb}$ \\
\hline 200 & “ & $4,93 \mathrm{Ba}$ & $5,33 \mathrm{Ba}$ & $7,12 \mathrm{Aa}$ \\
\hline \multicolumn{5}{|c|}{ Número de folhas } \\
\hline 0 & “ & $63,90 \mathrm{Bb}$ & $70,50 \mathrm{Aa}$ & $58,81 \mathrm{Ca}$ \\
\hline 200 & " & $73,00 \mathrm{Aa}$ & $67,50 \mathrm{Bb}$ & $53,25 \mathrm{Cb}$ \\
\hline \multicolumn{5}{|c|}{ Área foliar $\left(\mathrm{cm}^{2}\right)$} \\
\hline 0 & “ & $103,90 \mathrm{Ba}$ & $86,39 \mathrm{Cb}$ & $123,69 \mathrm{Aa}$ \\
\hline 200 & “ & $84,48 \mathrm{Bb}$ & $99,88 \mathrm{Aa}$ & $84,64 \mathrm{Bb}$ \\
\hline \multicolumn{5}{|c|}{ Massa seca folhas } \\
\hline 0 & “ & $10,51 \mathrm{Aa}$ & $10,18 \mathrm{Aa}$ & $9,13 \mathrm{Bb}$ \\
\hline 200 & “ & $10,55 \mathrm{Aa}$ & $9,35 \mathrm{Bb}$ & $9,97 \mathrm{~A} a b$ \\
\hline \multicolumn{5}{|c|}{ Massa seca ramos } \\
\hline 0 & “ & 13,93 Bb & $17,70 \mathrm{Aa}$ & $16,27 \mathrm{Ab}$ \\
\hline 200 & " & $20,78 \mathrm{Aa}$ & $19,21 \mathrm{Aa}$ & $18,98 \mathrm{Aa}$ \\
\hline
\end{tabular}

Médias seguidas das mesmas letras maiúsculas nas linhas e minúsculas nas colunas, para cada variável analisada, não diferem estatisticamente pelo Teste de Tukey ao nível de $5 \%$ de probabilidade. ns = não significativo

TABELA 2. Teor do óleo essencial ( $\mu \mathrm{L} \mathrm{g}^{-1}$ de massa seca foliar) de Pogostemon cablin aos 75 dias após aplicação de extrato de alga marinha e $200 \mathrm{mg} \mathrm{L}^{-1} \mathrm{GA}_{3}$. Curitiba-PR, 2007.

\begin{tabular}{cccc}
\hline \multirow{2}{*}{$\mathbf{G A}_{3}\left(\mathbf{m g ~ L}^{-1}\right)$} & \multicolumn{3}{c}{ Extrato de alga marinha $\left(\mathbf{m g ~ L}^{-1}\right)$} \\
\cline { 2 - 4 } & $\mathbf{0}$ & $\mathbf{1 5}$ & $\mathbf{3 0}$ \\
\hline 0 & $39,39 \mathrm{Cb}$ & $67,03 \mathrm{Aa}$ & $56,54 \mathrm{Ba}$ \\
200 & $60,01 \mathrm{Aa}$ & $60,71 \mathrm{Aa}$ & $23,88 \mathrm{Bb}$
\end{tabular}

Médias seguidas das mesmas letras maiúsculas nas linhas e minúsculas nas colunas, para cada variável analisada, não diferem estatisticamente pelo Teste de Tukey ao nível de $5 \%$ de probabilidade.

o menor teor encontrado em plantas tratadas com $\mathrm{GA}_{3}$ combinado a $30 \mathrm{mg} \mathrm{L}^{-1}$ pode estar relacionado ao aumento no número de ramos e a diminuição do número de folhas.

A produtividade do óleo essencial de patchouli também foi alterada após aplicação de reguladores vegetais, com aumento na produtividade quando utilizado $15 \mathrm{mg} \mathrm{L}^{-1}$ de extrato de alga marinha e quando utilizado somente $\mathrm{GA}_{3}$. Farooqi et al. (1993) observaram que quando aplicada as concentrações de 100, 150 e $200 \mathrm{mg} \mathrm{L}^{-1}$ de $\mathrm{GA}_{3}$ em plantas de Artemisia pallens Wall, somente o tratamento com a maior concentração foi superior na produtividade do óleo essencial quando comparado ao tratamento testemunha. Entretanto, a utilização de $30 \mathrm{mg} \mathrm{L}^{-1}$ de extrato de alga marinha e extrato de alga marinha combinado a $\mathrm{GA}_{3}$ tendem a diminuir a produtividade (Tabela 3 ).

A análise do óleo essencial revelou a presença dos seguintes sesquiterpenos: beta patchouleno, cariofileno, alfa guaieno, gama patchouleno, alfa patchouleno, seicheleno, beta guaieno, alfa selineno, alfa bulneseno, nor patchoulenol, pogostol e patchoulol (Tabela 4).

A composição do óleo essencial de patchouli foi afetada tanto pela fonte quanto pela concentração do regulador vegetal utilizado. De modo geral todos os reguladores utilizados parecem contribuir para o aumento do constituinte majoritário patchoulol. $\mathrm{O}$ efeito de reguladores vegetais na composição 
TABELA 3. Produtividade de óleo essencial $\left(\mathrm{L} \mathrm{ha}^{-1}\right)$ de Pogostemon cablin aos 75 dias após aplicação de extrato de alga marinha e $200 \mathrm{mg} \mathrm{L}^{-1} \mathrm{GA}_{3}$. Curitiba-PR, 2007.

\begin{tabular}{cccc}
\hline \multirow{2}{*}{$\mathbf{A A}_{3}\left(\mathbf{m g ~ L}^{-1}\right)$} & \multicolumn{3}{c}{ Extrato de alga marinha $\left(\mathbf{m g ~ L}^{-1}\right)$} \\
\cline { 2 - 4 } & $\mathbf{0}$ & $\mathbf{1 5}$ & $\mathbf{3 0}$ \\
\hline 0 & $4,23 \mathrm{Cb}$ & $10,73 \mathrm{Aa}$ & $8,00 \mathrm{Ba}$ \\
& $10,04 \mathrm{Aa}$ & $9,17 \mathrm{Bb}$ & $8,62 \mathrm{Cb}$
\end{tabular}

Médias seguidas das mesmas letras maiúsculas nas linhas e minúsculas nas colunas, para cada variável analisada, não diferem estatisticamente pelo Teste de Tukey ao nível de $5 \%$ de probabilidade.

TABELA 4. Composição do óleo essencial de Pogostemon cablin após 75 dias de cultivo, com aplicação de $\mathrm{GA}_{3}$ e extrato de alga marinha e $200 \mathrm{mg} \mathrm{L}^{-1} \mathrm{GA}_{3}$.

\begin{tabular}{|c|c|c|c|c|c|c|c|}
\hline \multirow{3}{*}{ Composto (\%) } & & \multicolumn{6}{|c|}{ Regulador Vegetal (mg L-1) } \\
\hline & $\mathrm{GA}_{3}$ & 0 & 0 & 0 & 200 & 200 & 200 \\
\hline & Extrato de alga marinha & $\mathbf{0}$ & 15 & 30 & 0 & 15 & 30 \\
\hline Beta patchouleno & & 1,47 & 1,19 & 1,13 & 1,18 & 1,28 & 1,23 \\
\hline Cariofileno & & 3,60 & 3,12 & 3,18 & 3,23 & 3,47 & 3,39 \\
\hline Alfa guaieno & & 9,33 & 7,76 & 7,74 & 8,13 & 8,84 & 8,86 \\
\hline Gama patchouleno & & 6,61 & 5,33 & 5,45 & 5,68 & 6,20 & 6,00 \\
\hline Alfa patchouleno & & 4,06 & 3,23 & 3,31 & 3,49 & 3,76 & 3,64 \\
\hline Seicheleno & & 1,65 & 1,37 & 1,38 & 1,42 & 1,53 & 1,53 \\
\hline Beta guaieno & & 1,06 & 0,80 & 0,92 & 0,93 & 1,02 & 0,89 \\
\hline Alfa selineno & & 2,03 & 1,66 & 1,72 & 1,80 & 1,93 & 1,90 \\
\hline Alfa bulneseno & & 13,07 & 10,97 & 11,29 & 11,86 & 12,8 & 12,41 \\
\hline Nor patchoulenol & & 1,06 & 1,28 & 1,19 & 1,19 & 1,10 & 1,15 \\
\hline Pogostol & & 2,61 & 3,30 & 3,25 & 3,00 & 2,96 & 3,09 \\
\hline Patchoulol & & 42,4 & 48,58 & 48,06 & 46,2 & 44,75 & 47,07 \\
\hline
\end{tabular}

do óleo essencial tem sido observado em outras espécies aromáticas. Em Artemisia annua L. a aplicação de $\mathrm{GA}_{3}$ resulta em aumento dos teores de artemisina (Farooqi et al., 1996), e a aplicação da citocinina 6-benzilaminopurina altera a composição do óleo essencial de Mentha x piperita (Scavroni et al., 2006). Logo, as alterações na composição do óleo essencial de patchouli frente à aplicação de reguladores vegetais estão de acordo com a literatura pesquisada.

Segundo Deguerry et al. (2006), os componentes do óleo essencial do patchouli são formados a partir do farnesil-difosfato que sofre alterações estruturais dando origem a 2 intermediários humulil cátion e E,E - germacradienil cátion, este último é o responsável pela síntese do pogostol e do patchoulol, compostos majoritários no óleo essencial desta espécie.

Tanto a síntese do patchoulol quanto do pogostol ocorrem em presença de água, entretanto, na ausência de água os mesmos intermediários que dão origem a esses compostos originam o seicheleno e o alfa guaieno, alfa buleseno e guaia4,11-dieno, respectivamente. Nestas reações, ocorre desprotonização e a molécula de água não é utilizada como substrato, sabendo-se que a síntese destes não é dependente da quebra de uma molécula de água, sugere-se que a concentração destes constituintes no óleo essencial é diretamente dependente do balanço dos constituintes patchoulol e pogostol, tendo em vista a presença de precursores comuns. A biossíntese de patchoulol e seicheleno possui como precursor comum um intermediário da rota metabólica. Entretanto, na síntese de pogostol, alfa guaieno e alfa buleseno o precursor comum é o guaianil cátion.

Deste modo, a maior produção de patchoulol nos tratamentos com reguladores vegetais pode estar relacionada à menor síntese de compostos minoritários no óleo essencial tais como beta patchouleno, alfa patchouleno e gama patchouleno nestes tratamentos, os quais são intermediários da síntese do patchoulol e competem com este na formação de substratos. Sugere-se que a aplicação 
de reguladores vegetais direciona a rota de síntese destes compostos para a formação dos compostos majoritários do óleo essencial de patchouli. O processo semelhante ocorre com a maior síntese de pogostol e menor síntese de alfa guaieno quando houve aplicação de giberelina e extrato de alga marinha. Deste modo, futuras avaliações bioquímicas poderão ser utilizadas para investigar essa hipótese.

\section{CONCLUSÕES}

Nas condições em que foi conduzido o experimento pode-se concluir que:

$\mathrm{GA}_{3}$ promove aumento na altura das plantas de patchouli.

A utilização de extrato de alga marinha combinado a giberelina não altera a altura das plantas; entretanto, promove diferenças no número de ramos, número de folhas e área foliar.

A aplicação de extrato de alga marinha na concentração de $15 \mathrm{mg} \mathrm{L}^{-1}$ resulta em maior teor de óleo essencial, o qual também pode ser obtido com aplicação de $200 \mathrm{mg} \mathrm{L}^{-1}$ de $\mathrm{GA}_{3}$.

$\mathrm{O}$ extrato de alga marinha e $\circ \mathrm{GA}_{3}$ não devem ser aplicados simultaneamente em plantas de patchouli.

A aplicação foliar de extrato de alga marinha e giberelina alteram a composição do óleo essencial de Pogostemon cablin, promovendo maior concentração de patchoulol e pogostol neste.

\section{AGRADECIMENTOS}

Os autores agradecem a Capes pela bolsa concedida e a empresa Hérbia Óleos Essenciais Ltda. pelos recursos financeiros destinados ao trabalho.

\section{REFERÊNCIA}

CHAUDHRY, N.Y.; KHAN, A.S. Effect of growth hormones i.e., $\mathrm{GA}_{3}$, IAA and kinetin on 1. Length and diameter of shoot, 2. Early Initiation of cambium and maturation of metaxylen elements in Cicer arietinum L. Journal of Biological Sciences, v.3, n.11, p.1263-6, 2000.

$\mathrm{CROUCH}$, I.J.; VAN STADEN, J. Evidence for the presence of plant growth regulators in commercial seaweed products. Journal of plant growth regulation, v.13, n.1, p.21-9, 1993.

DEGUERRY, F. et al. The diverse sesquiterpene profile of patchouli, Pogostemon cablin, is correlated with a limited number of sesquiterpene synthases. Archives of Biochemistry and Biophysics, v.37, p.123-36, 2006.

EPAGRI. Normas técnicas para cultivo de capimlimão, citronela, palma-rosa e patchuli, Florianópolis, 2004. 58p. (Epagri. Sistemas de Produção, 37).

ERGÜN, N.; TOPCUOGLU, F.; YILDIZ, A. Auxin (indole3-acetic acid), gibberellic acid $\left(\mathrm{GA}_{3}\right)$, abscisic acid (ABA) and cytokinin (zeatin) production by some species of mosses and lichens. Turk Journal Botany, v. 26, p.13-8, 2002.

FAROOQI, A.A.; DEVIAH, K. A; VASUNDRA, M. Effect of some growth regulators and pinching on growth, yield and essential oil content of davana (Artemisia pallens Wall.) Indian Perfumer, v. 37, p.19-23, 1993.

FAROOQI, A.H.; SHUKLA, A.; KHAN, A. Effect of plant age and $\mathrm{GA}_{3}$ on artemisinin and essential oil yield in Artemisia annua L. Journal of Herbs, Spices \& Medicinal Plants, v. 4, n.1, p.73-80, 1996.

HENDERSON, W. et al. Chemical and morphological studies on sites of sesquiterpene accumulation in Pogostemon cablin (Patchouli). Phytochemistry, v.9, p.1219-28, 1970.

HU L.F.; LI, S.P.; CAOB, H.; LIU, J.J.; GAO J.L.; YANG, F.Q.; WANG, Y.T. GC-MS fingerprint of Pogostemon cablin in China. Journal of Pharmaceutical and Biomedical Analysis, v. 42, p.1-7, 2005.

RAIJ, B. et al. Recomendações de adubação e calagem para o Estado de São Paulo. Instituto Agronômico e Fundação IAC, Campinas, 1996. 285p.

SCAVRONI, J. et al. Rendimento e composição química do óleo essencial de Mentha piperita L. submetida a aplicações de giberilina e citocinina. Revista Brasileira de Horticultura, v. 8, n. 4, p. 40-3, 2006.

SHUKLA, A.; FAROOQI, A.H.A.E. Review article: Ultilization of plant growth regulators in aromatic plant production. Current Research Medicinal \& Aromatic Plants, v.12, n. 3, p. 152-7, 1990.

SILVA, F.A.S.; AZEVEDO, C.A.V. A new version of the assistat-statistical assistance software. In: WORLD CONGRESS ON COMPUTERS IN AGRICULTURE, 4., 2006, Orlando. American Society of Agricultural Engineers. Orlando, 2006. p. 393-6.

STOEVA, T.; ILIEV, L. Influence of some phenylurea cytokinins on spearmint essential oil composition. Bulgarian Journal Plant Physiology, v. 23, n. 3, p.66-71, 1997.

ZHANG, X.; ERVIN, E.H. Cytokinin containing seaweed and humic acid extracts associated with creeping. Crop Science, v. 44, p.1-10, 2004. 